IMMUNOLOGY CORNER

\title{
Recurrent Pneumonia and Immune Deficiency
}

\author{
Sagar Bhattad \\ Pediatric Infectious Disease (2020): 10.5005/jp-journals-10081-1285
}

Recurrent lower respiratory tract infection (pneumonia) is one of the commonest presentations of an underlying immune deficiency. As such, in all children and adults presenting with recurrent pneumonia, one must think of a primary immune deficiency (PID). Kindly note, PIDs are better known as inborn errors of immunity (IEI).

When faced with a child with recurrent pneumonia, the following differentials are often considered:

- Congenital heart disease (left to right shunts, e.g., ventricular septal defect).

- Aspiration syndrome [e.g., gastroesophageal reflux (GER) disease].

- Congenital lung malformation (e.g., congenital lobar emphysema).

- Cystic fibrosis and ciliary dyskinesia.

- Human immunodeficiency virus (HIV) infection.

However, the list is incomplete without the mention of PID/IEI. In the next section, we will discuss a few cases and understand how children and adults presenting with recurrent respiratory issues must be evaluated for a possible immune defect.

\section{Case Descriptions}

\section{Case 1}

A 3-year-old boy presented with the 4th episode of pneumonia. He was hospitalized for 7-10 days during each of these episodes, treated with antimicrobials, and received oxygen therapy. He was well in-between these episodes.

He had been investigated extensively-

- Echocardiography: normal.

- Computed tomography (CT) chest: no anomaly.

- Sweat chloride test: normal.

- Nuclear scan for GER: normal (for GER).

- Human immunodeficiency virus rapid test: non-reactive.

He was referred to pediatric immunology services.

On a detailed clinical examination, tonsils were absent (Fig. 1). Lymph nodes were not palpable (Note: lymphoid growth is at its peak during 2-8 years of life).

\section{Investigations}

Complete blood counts (CBC)—no neutropenia or lymphopenia.

He was investigated further-

Immunoglobulins-

$\lg G<130 \mathrm{mg} / \mathrm{dL}(345-1,236)$.

$\lg A<30 \mathrm{mg} / \mathrm{dL}(14-159)$.

$\lg M<20 \mathrm{mg} / \mathrm{dL}$ (43-207).

All the immunoglobulins were low!

Next step: B cell counts were performed.

B cells (CD19): $0.5 \%$ (N 10-15\%).

$B$ cells were absent

Department of Pediatrics, Aster CMI Hospital, Bengaluru, Karnataka, India

Corresponding Author: Sagar Bhattad, Department of Pediatrics, Aster CMI Hospital, Bengaluru, Karnataka, India, Phone: +91 9779433934, e-mail: drsagarbhattad@gmail.com

How to cite this article: Bhattad S. Recurrent Pneumonia and Immune Deficiency. Pediatr Inf Dis 2020;2(4):161-162.

Source of support: Nil

Conflict of interest: None

\section{Database}

3-year-old boy with recurrent pneumonia $\downarrow$

Absent tonsils and lymph nodes

$$
\downarrow
$$

Low immunoglobulins and absent B cells $\downarrow$

Diagnosis: X-linked Agammaglobulinemia

Message: Recurrent pneumonia-serum immunoglobulins must be tested.

\section{Case 2}

A 28-year-old man was referred by the pulmonologist for immunological evaluation. He had had repeated episodes of pneumonia requiring 7-10 days of antimicrobials during each episode. He had 2-3 such episodes every year for the past 8 years.

Past history: Repeated episodes of ear discharge from the age of 10. Recurrent sinusitis from the age of $15-3$ to 4 episodes/year (was said to have allergic rhinitis!).

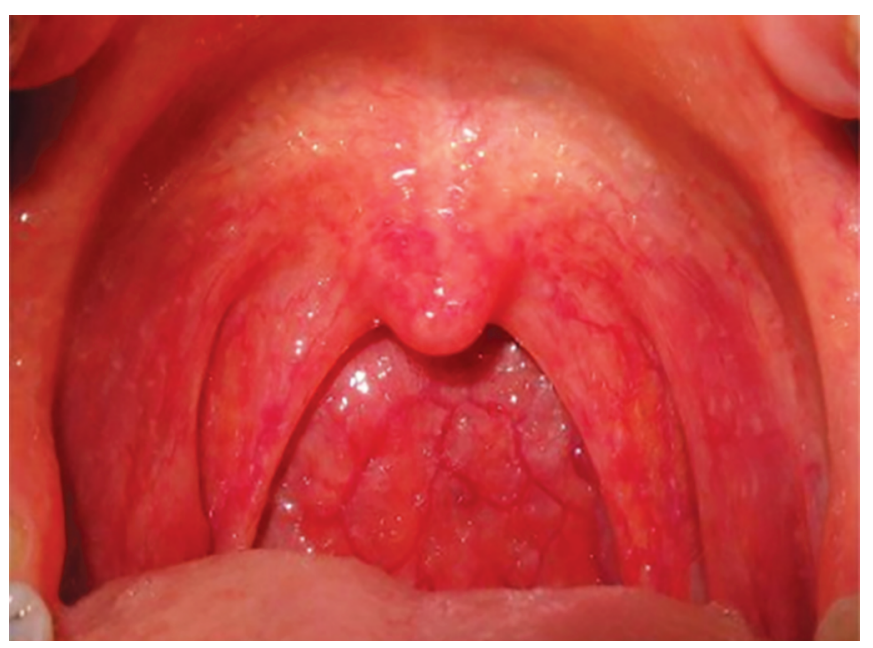

Fig. 1: Absent tonsils in a boy with X-linked agammaglobulinemia

(c) The Author(s). 2020 Open Access This article is distributed under the terms of the Creative Commons Attribution 4.0 International License (https:// creativecommons.org/licenses/by-nc/4.0/), which permits unrestricted use, distribution, and non-commercial reproduction in any medium, provided you give appropriate credit to the original author(s) and the source, provide a link to the Creative Commons license, and indicate if changes were made. The Creative Commons Public Domain Dedication waiver (http://creativecommons.org/publicdomain/zero/1.0/) applies to the data made available in this article, unless otherwise stated. 
He had been extensively evaluated before being referred.

Computed tomography chest done thrice revealed consolidation involving different lobes during these episodes of pneumonia. The latest CT chest showed changes of bronchiectasis in the lower lobes. He had undergone bronchoscopies but no definite diagnosis was offered. He had been treated with antitubercular therapy twice empirically! Human immunodeficiency virus had been tested negative.

We looked at the immunoglobulin profile:

$\lg G 70 \mathrm{mg} / \mathrm{dL}(639-1,349)$.

$\lg A<26 \mathrm{mg} / \mathrm{dL}(70-312)$.

$\lg \mathrm{M}<20 \mathrm{mg} / \mathrm{dL}(56-352)$.

He had panhypogammaglobulinemia.

B cell counts: $15 \%$ (8-20\%) (B cells were normal).

\section{Database}

28-year-old man with recurrent pneumonia and sinusitis

$$
\downarrow
$$

HIV negative

$$
\downarrow
$$

Hypogammaglobulinemia and normal B cell counts

$$
\downarrow
$$

Diagnosis: Common Variable Immune Deficiency

Message: Recurrent pneumonia/rhinosinusitis in adults-look for immunoglobulins.

\section{Persistent Pneumonia/Non-resolving Pneumonia}

A 5-year-old child was hospitalized with severe pneumonia and was being ventilated. No improvement was noted after 3 weeks of antibiotics (1st and 2nd line). Contrast-enhanced CT (CECT) of the chest showed multiple nodules in the lung parenchyma.

For a definitive diagnosis, lung aspirate was performed and smears showed Aspergillus!

\section{Diagnosis: Aspergillus pneumonia}

Aspergillus is an unusual organism and infection with such organism(s) warrants evaluation for immune deficiency.

Evaluation showed-

Complete blood counts: no neutropenia.

IgG: 2,300 mg/dL (345-1,236), IgA: $220 \mathrm{mg} / \mathrm{dL}$ (14-159), IgM: $150 \mathrm{mg} / \mathrm{dL}(43-207)$.
Nitroblue tetrazolium (NBT) dye reduction test is a test to look for a respiratory burst in neutrophils and is a screening test for chronic granulomatous disease (CGD). Nitroblue tetrazolium was abnormal in the index case.

\section{Diagnosis: Chronic Granulomatous Disease \\ Database}

Non-resolving pneumonia

$$
\downarrow
$$

Immunoglobulins-elevated

$\downarrow$

NBT test-abnormal (Note: DHR is a better test in this setting). $\downarrow$

Chronic granulomatous disease (DHR—dihydrorhodamine test).

- Message: Non-resolving pneumonia-

- Investigate for an underlying organism.

- Immune deficiency (phagocytic defect) likely.

- Aspergillus pneumonia in a non-neutropenic setting-always evaluate for CGD. ${ }^{1-3}$

\section{Conclusion}

Children and adults presenting with recurrent and/or persistent pneumonia, pneumonia caused by unusual organisms must be investigated for a possible immune deficiency. B cell defects commonly present with recurrent sinopulmonary infections. As such, estimation of serum immunoglobulins is an important and cost-effective test while evaluating these patients.

\section{References}

1. Reisi M, Azizi G, Kiaee F, et al. Evaluation of pulmonary complications in patients with primary immunodeficiency disorders. Eur Ann Allergy Clin Immunol 2017;49(3):122-128.

2. Yazdani R, Abolhassani $H$, Asgardoon $M H$, et al. Infectious and noninfectious pulmonary complications in patients with primary immunodeficiency disorders. J Investig Allergol Clin Immunol 2017;27(4):213-224. DOI: 10.18176/jiaci.0166.

3. Jesenak M, Banovcin $P$, Jesenakova B, et al. Pulmonary manifestations of primary immunodeficiency disorders in children. Front Pediatr. 2014;2:77. DOI: 10.3389/fped.2014.00077. 